\title{
Drift Waves in General Toroidal Geometry
}

\author{
J.L.V. Lewandowski \\ Princeton Plasma Physics Laboratory, \\ Princeton University, P.O. Box 451, Princeton NJ 08543
}

(January 31, 2000)

\begin{abstract}
A model, based on gyro-kinetic ions and fluid electrons, to study drift waves in low $-\beta[\beta=$ (kinetic pressure $) /($ magnetic pressure $)]$ stellarator plasmas is presented. The model equations are written in straight-field-line coordinates and are valid for arbitrary, fully three-dimensional configurations with closed, nested magnetic surfaces. An implicit method, coupled with a subcycling technique for the electrons, is used to solve the time-dependent, along-thefield-line equations. Numerical calculations are carried out for a 3-field-period toroidal heliac. The geometrical effects that enter the model equations are calculated and displayed in physical space using advanced visualization techniques.
\end{abstract}

Pacs \# : 52.35Kt, 52.30Jb, 52.35Ra 


\section{INTRODUCTION}

It is now generally accepted in the fusion community that even if fast, large-scale magnetohydrodynamic (MHD) instabilities can be suppressed, magnetically-confined plasmas always contain sufficient free energy to drive slow, short-scale instabilities. These slow, short-scale instabilities, often called microinstabilities ${ }^{1,2}$, are a major concern as confinement is concerned. The cross-field (perpendicular) transport associated with microinstabilities is often one to two orders magnitude larger than the neoclassical transport, and it is called 'anomalous' for this reason ${ }^{3,4}$.

Substantial efforts have been made to understand microinstability dynamics and the associated transport in tokamak geometry. However very little work has been published on microinstabilities in stellarator geometry. The earlier work of Bhattacharjee et al. considered an electron drift wave model (with cold ions) in helically symmetric configurations; the main conclusion of the paper by Bhattacharjee et al. is that localized and extended modes can coexist in such configurations ${ }^{5}$. The spectrum of the model used by Bhattacharjee et al. has been calculated by Persson et al. ${ }^{6}$. The first drift wave calculations in realistic stellarator geometry were carried out by Dominguez and co-workers ${ }^{7}$ for the dissipative trapped electron mode (DTEM); they showed that extended modes as well as strongly localized modes do exist (in the linear approximation) in a fully three-dimensional configuration.

In this paper, we present calculations of the ion collisionless gyro-kinetic equation in the linear regime for a low- $\beta$ stellarator plasma. Braginskii's fluid equations ${ }^{8}$ are used to determine the electron dynamics. The perturbed ion density is obtained by a direct integration over velocity space of the ion distribution function, while the perturbed electron density is determined from the electron continuity equation. The closure relation for the perturbed electrostatic potential is the quasineutrality condition. We use the ballooning representation $^{9,10}$ for fluctuating quantities and the equations are written in straight-field-line coor- 
dinates $^{11}$. Our initial-value code is convenient for studying the Ion-Temperature-Gradient driven (ITG) mode ${ }^{12}$ in general 3-D plasmas. Since the model presented here is valid for fully 3-D (non-axisymmetric) plasmas, it can be applied to stellarator configurations as well as tokamaks with field coil ripple effects.

This paper is organized as follows. In section 2, the collisionless ion gyro-kinetic equation in the ballooning representation is written in a form convenient for direct numerical integration. In section 3, the evolution equation for the perturbed electron density, based on a fluid model (Appendix A), is given. The system of equations describing the fluctuating ion distribution function and the perturbed electron density is coupled through the quasineutrality condition, and can be solved using standard numerical techniques (section 4 and Appendix B). In section 4, we calculate the critical ion temperature gradient parameter $\left(\eta_{\text {ic }}\right)$ and the $k_{\perp}$ spectrum of the ITG mode in a low- $\beta$, low-shear stellarator plasma. The conclusion is given in section 6 .

\section{ION DYNAMICS}

The Ion-Temperature-Gradient-Driven (ITG) mode is a drift-type instability which arises from the free energy stored in the ion pressure gradient. In slab geometry and for a flat density profile, the mode is attributed to the coupling between the electron drift wave and the ion acoustic waves. Rudakov and Sagdeev considered a simple ITG model ${ }^{12}$ in slab geometry and for a flat density profile, neglecting kinetic effects. The inclusion of magnetic shear and kinetic effects were investigated by Pogutse ${ }^{13}$. Using a fluid model and assuming an adiabatic response for the electrons, a systematic study of the mode structure of the ITG mode in sheared slab geometry was carried out by Coppi et al. ${ }^{14}$, and by Hassam et al. ${ }^{15}$.

As shown by Horton et al. ${ }^{16}$, the ITG mode in toroidal geometry is mainly driven by unfavorable magnetic curvature rather than the coupling of the electron drift wave to the ion 
acoustic waves. It is worth noting that other pressure-gradient-driven modes, such as the collisionless trapped-particle mode ${ }^{17}$, the trapped-electron mode ${ }^{18}$ and the residual trappedion mode ${ }^{19}$, can be driven unstable in the presence of unfavorable magnetic curvature.

The confining magnetic field is written in straight-field-line coordinates ${ }^{11}$

$$
\mathbf{B}=\nabla \alpha \times \nabla \psi
$$

where $\alpha \equiv \zeta-q(s) \theta$ is the field line label, $2 \pi \psi$ is the enclosed poloidal flux, $\theta(\zeta)$ is the poloidal (toroidal) angle-like coordinate (with period $2 \pi$ ), $s \equiv \Psi_{\mathrm{T}} / \Psi_{\mathrm{T}}^{(b)}$ is the normalized radial label, $\Psi_{\mathrm{T}}$ is the enclosed toroidal flux within the magnetic surface, and $\Psi_{\mathrm{T}}^{(b)}$ is $\Psi_{\mathrm{T}}$ evaluated at the plasma boundary. The equilibrium is computed using $s$ as a radial label, but it is convenient to use the effective local minor radius $\rho \equiv \bar{a} \sqrt{s}$ (where $\bar{a}$ is the average minor radius of the last closed magnetic surface) in the drift-wave calculations. For drift-type $\left(k_{\|} / k_{\perp} \ll 1\right)$ modes, it is convenient to use the ballooning representation ${ }^{9,10}$ for fluctuating quantities. Letting $F=F_{0}$ (equilibrium) $+\delta F$ (fluctuation) and assuming $\left|\delta F / F_{0}\right| \ll 1$, one writes $^{9,10}$

$$
\widetilde{F} \equiv \frac{\delta F}{F_{0}}=\widehat{F} \exp \left(i \epsilon^{-1} S\right)
$$

where the amplitude $\widehat{F}$ varies on the equilibrium scale length; we demand that the eikonal $S$ satisfies $\mathbf{B} \cdot \boldsymbol{\nabla} S=0$ to all orders in the expansion parameter $\epsilon \ll 1$. The spatial variation of $\widehat{F}$ represents the deviation from flute-like modes. One can use the extended toroidal angle $\bar{\zeta}=[-\infty,+\infty]$ as a label along the field line and the amplitude in Eq. (2) is written as $\widehat{F}=\widehat{F}(\bar{\zeta}, t)$. To recover the standard form of the normal mode analysis, we make the transformation $\widehat{F}(\bar{\zeta}, t) \mapsto \widehat{F}(\bar{\zeta}) \exp (-i \omega t)$, where $\omega$ is the normal mode frequency. The requirement $\mathbf{B} \cdot \boldsymbol{\nabla} S=0$ implies that $S=S(\alpha, q)$ where here $q$ is used as a radial coordinate instead of $s, \rho$ or $\psi$. It is natural to introduce the lowest-order perpendicular wavevector $\operatorname{as}^{10}$

$$
\mathbf{k}_{\perp} \equiv \epsilon^{-1} \nabla S=N\left[\boldsymbol{\nabla} \zeta-q \boldsymbol{\nabla} \theta-\dot{q}\left(\theta-\theta_{k}\right) \boldsymbol{\nabla} \rho\right]
$$


where $N \equiv(\partial S / \partial \alpha) / \epsilon \gg 1$ is the toroidal mode number, a dot denotes a derivative with respect to $\rho$, and $\theta_{k} \equiv(\partial S / \partial q)(\partial S / \partial \alpha)^{-1}$ is the radial mode number. The equilibrium distribution for the ions must be independent of the gyro-angle and constant along the magnetic field line ${ }^{20,10}$; these requirements are fulfilled for a Maxwellian distribution

$$
F_{M}=\frac{n_{0}}{\pi^{3 / 2} v_{\mathrm{thi}^{3}}} \exp \left(-\frac{v^{2}}{v_{\mathrm{thi}}^{2}}\right)
$$

where $v_{\text {thi }}=\sqrt{2 T_{\mathrm{i} 0}(\rho) / m_{\mathrm{i}}}$ is the ion thermal velocity. The perturbed ion distribution function can be written as

$$
\delta f=-\frac{F_{M}}{\tau} \widetilde{\Phi}+\left(h+\frac{J_{0}}{\tau} \widetilde{\Phi} F_{M}\right) J_{0}
$$

where the nonadiabatic part $h$ satisfies the linear, collisionless gyro-kinetic equation ${ }^{10}$

$$
\frac{\partial h}{\partial t}=-v_{\|} \nabla_{\|}\left(h+\frac{\widetilde{\Phi}}{\tau} J_{0} F_{M}\right)-i \omega_{\mathrm{di}} h+i\left(\omega_{\star \mathrm{i}}^{T}-\omega_{\mathrm{di}}\right) \frac{\widetilde{\Phi}}{\tau} J_{0} F_{M}
$$

In Eqs $(5,6) \tau \equiv T_{\mathrm{i} 0} / T_{\mathrm{e} 0}, J_{0}$ is the zeroth-order Bessel function (arising from the averaging over the fast gyro-motion) with argument $\xi_{\perp} \equiv k_{\perp} v_{\perp} / \omega_{\text {ci }}, k_{\perp}=\sqrt{\mathbf{k}_{\perp} \cdot \mathbf{k}_{\perp}}$ is the magnitude of the lowest-order perpendicular wavevector and $\omega_{\mathrm{ci}} \equiv e B /\left(m_{\mathrm{i}} c\right)$ is the ion cyclotron frequency. Note that $\xi_{\perp}$ depends on the perpendicular velocity as well as the position along the field line through the dependence of $B$ and $k_{\perp} \propto|\nabla S(\mathbf{r})|$. The effect of the global magnetic shear manifests itself in the secular behavior of $k_{\perp}$. The first term on the right-hand side of Eq. (5) is the adiabatic part of the perturbed ion distribution function, while the last term arises because the guiding center density and the particle density do not coincide due to the finite ion Larmor radius (polarization term). In Eq. (6)

$$
\omega_{\star \mathrm{i}}^{T} \equiv \frac{c T_{\mathrm{i} 0}}{e B}\left(\mathbf{k}_{\perp} \times \widehat{\mathbf{e}}_{\|}\right) \cdot \frac{\nabla F_{M}}{F_{M}},
$$

is the velocity-dependent ion diamagnetic drift frequency and

$$
\omega_{\mathrm{di}} \equiv \omega_{\mathrm{ci}}{ }^{-1}\left[\widehat{\mathbf{e}}_{\|} \times\left(v_{\|}^{2} \boldsymbol{\kappa}+\frac{v_{\perp}^{2}}{2} \frac{\boldsymbol{\nabla} B}{B}\right)\right] \cdot \mathbf{k}_{\perp},
$$

is the velocity-dependent ion curvature drift frequency, $\widehat{\mathbf{e}}_{\|} \equiv \mathbf{B} / B$ is the unit vector along $\mathbf{B}, \boldsymbol{\kappa} \equiv\left(\widehat{\mathbf{e}}_{\|} \cdot \boldsymbol{\nabla}\right) \widehat{\mathbf{e}}_{\|}$is the magnetic curvature and $\nabla_{\|} \equiv\left(\widehat{\mathbf{e}}_{\|} \cdot \nabla\right)_{\alpha}$ is the parallel gradient 
operator keeping the field line label, $\alpha$, constant. Taking into account that $\mathbf{B} \cdot \boldsymbol{\nabla} F_{M}=0$ (since $n_{0}$ and $T_{\mathrm{i} 0}$ are flux surface quantities) and that

$$
\frac{F_{M}^{-1} \partial F_{M} / \partial t}{h^{-1} \partial h / \partial t} \sim \frac{\omega_{0}}{\omega} \ll 1
$$

where $2 \pi / \omega$ is the typical drift-wave timescale and $2 \pi / \omega_{0}$ is the transport timescale, it is convenient to introduce $g\left(\bar{\zeta}, V_{\|}, V_{\perp} ; t\right) \equiv h / F_{M}$ and the gyro-kinetic equation becomes

$$
\frac{\partial g}{\partial t^{\prime \prime}}=-\Omega_{\|} V_{\|} \frac{\partial}{\partial \bar{\zeta}}(g+\widetilde{\phi})-i \Omega_{\mathrm{di}} g+i\left(\Omega_{\star \mathrm{i}}^{T}-\Omega_{\mathrm{di}}\right) \widetilde{\phi}
$$

where $t^{\prime \prime} \equiv \omega_{\star} t$ is the normalized time, $\widetilde{\phi}\left(V_{\|}, V_{\perp}, \bar{\zeta}, t\right) \equiv J_{0} F_{M} \widetilde{\Phi} / \tau$ is a generalized electrostatic potential,

$$
\Omega_{\star \mathrm{i}}^{T}\left(V_{\|}, V_{\perp}\right)=\tau \sqrt{b_{0}} S_{\star}\left[1+\eta_{\mathrm{i}}\left(V^{2}-\frac{3}{2}\right)\right]
$$

is the normalized ion diamagnetic drift frequency and

$$
\Omega_{\mathrm{di}}\left(\bar{\zeta}, V_{\|}, V_{\perp}\right)=2 \sqrt{b_{0}} \tau S_{\perp}\left(V_{\|}^{2}+V_{\perp}^{2} / 2\right)
$$

is the normalized ion curvature drift frequency. Here $V_{\|}\left(V_{\perp}\right)=v_{\|}\left(v_{\perp}\right) / v_{\text {thi }}$ is the normalized parallel (perpendicular) velocity; $b_{0} \equiv\left(k_{\theta} \rho_{s 0}\right)^{2}$ is a parameter (proportional to the square of the toroidal mode number $N), \rho_{s 0}=c_{s} / \omega_{\text {cio }}$ with $\omega_{\text {cio }}$ the ion cyclotron frequency evaluated at the magnetic axis, $\omega_{\star}(\rho) \equiv c_{s} / L_{n}$ is the electron drift frequency; $\eta_{\mathrm{i}} \equiv L_{n} / L_{T_{i}}$ is the ion temperature gradient parameter; $L_{n}(\rho) \equiv-\left(n_{0}^{-1} d n_{0} / d \rho\right)^{-1}$ is the equilibrium density scale length; $L_{T_{i}}(\rho) \equiv-\left(T_{\mathrm{i} 0}^{-1} d T_{\mathrm{i} 0} / d \rho\right)^{-1}$ is the equilibrium ion temperature scale length; $\Omega_{\|}(\bar{\zeta}) \equiv \sqrt{2 \tau} \epsilon_{n} S_{\|}$where $S_{\|}$describes the modulation of $B$ along the field line in the definition of the parallel gradient operator

$$
\left.\nabla_{\|} \equiv \widehat{\mathbf{e}}_{\|} \cdot \nabla\right|_{\alpha}=\frac{S_{\|}(\bar{\zeta})}{\bar{R}} \frac{\partial}{\partial \bar{\zeta}}
$$

We have also defined the nondimensional geometrical quantities

$$
S_{\star} \equiv \frac{\nabla \rho}{B_{\star}} \cdot\left(\widehat{\mathbf{e}}_{\|} \times \widehat{\mathbf{e}}_{\perp}\right)
$$


and

$$
S_{\perp}(\bar{\zeta}) \equiv \frac{\widehat{\mathbf{e}}_{\perp}}{B_{\star}} \cdot\left(\widehat{\mathbf{e}}_{\|} \times \mathbf{Q}\right)
$$

Here $\mathbf{Q} \equiv L_{n} \boldsymbol{\nabla} B / B=\mathcal{O} \sim \epsilon_{n} \equiv L_{n} / \bar{R}, \bar{R}$ is the average radius of the magnetic axis, $\widehat{\mathbf{e}}_{\perp} \equiv$ $\mathbf{k}_{\perp} / k_{\theta}, k_{\theta}=N q / \rho$ is the characteristic perpendicular wavevector and $B_{\star}(\bar{\zeta})=B / B_{0}=\mathcal{O}(1)$ is the magnetic field strength normalized to its value at the magnetic axis. It can be shown that the argument of the Bessel function, $\xi_{\perp}$, and the FLR parameter, $b$, can be written as

$$
b(\bar{\zeta})=b_{0} \tau L_{\perp}(\bar{\zeta}) \text { and } \xi_{\perp}\left(\bar{\zeta}, V_{\perp}\right)=\left[2 b_{0} \tau L_{\perp}(\bar{\zeta})\right]^{1 / 2} V_{\perp}
$$

where

$$
L_{\perp}(\bar{\zeta}) \equiv \frac{\widehat{\mathbf{e}}_{\perp} \cdot \widehat{\mathbf{e}}_{\perp}}{B_{\star}^{2}} \propto|\nabla \alpha|^{2} / B^{2}
$$

displays a secular (parabolic) behavior in $\bar{\zeta}$ when the global magnetic shear $\widehat{s} \equiv \rho q^{-1}(d q / d \rho)$ does not vanish. Using the relation ${ }^{21}$

$$
\int_{0}^{\infty} \exp (-\alpha x) J_{\lambda}(2 \beta \sqrt{x}) J_{\lambda}(2 \gamma \sqrt{x}) d x=\frac{1}{\alpha} I_{\lambda}(2 \beta \gamma / \alpha) \exp \left(-\frac{\beta^{2}+\gamma^{2}}{\alpha}\right),
$$

one can calculate the perturbed ion density by taking the velocity moment of Eq. (5)

$$
\begin{aligned}
\widetilde{n}_{\mathrm{i}}(\bar{\zeta}, t) \equiv \frac{\delta n_{\mathrm{i}}}{n_{0}} & =G(b) \frac{\widetilde{\Phi}(\bar{\zeta} ; t)}{\tau}+\frac{2}{\sqrt{\pi}} \int_{0}^{\infty} d V_{\perp} \int_{-\infty}^{+\infty} d V_{\|} V_{\perp} J_{0}\left(\bar{\zeta}, V_{\perp}\right) \\
& \times g\left(\bar{\zeta}, V_{\|}, V_{\perp} ; t\right) \exp \left(-V_{\|}^{2}-V_{\perp}^{2}\right)
\end{aligned}
$$

where $G(b) \equiv I_{0}(b) \exp (-b)$ and $I_{0}$ is the modified Bessel function. In the long-wavelength limit, one notes that $G(b) \approx b \ll 1$. We use the (normalized) perturbed ion density (19) in the quasineutrality condition $\left(\widetilde{n}_{\mathrm{i}} \simeq \widetilde{n}_{\mathrm{e}}\right)$ to obtain an equation for the (normalized) fluctuating electrostatic potential

$$
\widetilde{\Phi}=\frac{\tau}{G(b)}\left(\widetilde{n}_{\mathrm{e}}-\overline{\bar{n}}\right)
$$

where

$$
\overline{\bar{n}}(\bar{\zeta} ; t) \equiv \frac{2}{\sqrt{\pi}} \int_{0}^{\infty} d V_{\perp} \int_{-\infty}^{+\infty} d V_{\|} V_{\perp} J_{0}\left(\bar{\zeta}, V_{\perp}\right) g\left(\bar{\zeta}, V_{\|}, V_{\perp} ; t\right) \exp \left(-V_{\|}^{2}-V_{\perp}^{2}\right)
$$


can be calculated numerically once $g$ is known. If we assume that the electron response is adiabatic, $\widetilde{n}_{\mathrm{e}}=\widetilde{\Phi}$, Eq. (20) then reduces to

$$
\widetilde{\Phi}=\frac{\overline{\bar{n}}}{1-G(b) / \tau} .
$$

\section{ELECTRON DYNAMICS}

The perturbed electron density, which is yet unspecified, is required in the quasineutrality condition (20). One possible model is to simply assume that the transport along the field line is large enough to short-circuit charge separation. In this case, the electron response is adiabatic and the electrostatic potential can be calculated directly from Eq. (22). However in low- $T_{\mathrm{e}}$, high-density edge plasmas ion-electron collisions can prevent the electron density perturbation from reaching a Boltzmann distribution. Assuming that the parallel wavelength

of the mode $\left(\sim k_{\|}^{-1}\right)$ is larger than the electron mean free path, the (normalized) perturbed electron density can be calculated from Braginskii's fluid equations ${ }^{8}$ (appendix A)

$$
\frac{\partial \widetilde{n}_{\mathrm{e}}}{\partial t^{\prime \prime}}=\xi_{\mathrm{c}}\left[L_{n}^{2} \nabla_{\|}^{2} \widetilde{h}-Q_{\|}(\bar{\zeta}) L_{n} \nabla_{\|} \tilde{h}\right]+i \sqrt{b_{0}}\left[\widetilde{\Phi}+2 S_{\perp}(\bar{\zeta}) \widetilde{h}\right]-S_{\mathrm{i}}^{(\|)}
$$

where $\widetilde{h} \equiv \widetilde{n}_{\mathrm{e}}-\widetilde{\Phi} \simeq \widetilde{n}_{\mathrm{i}}-\widetilde{\Phi}$ and $\xi_{\mathrm{c}} \gg 1$ is related to the plasma collisionality. The first term on the right-hand side of Eq. (23) describes the transport of current density along the magnetic field line; the second term represents the transport of current density across the magnetic field; and finally the third term [which can be calculated from the velocity moment of $\delta f$; see Appendix $\mathrm{A}]$ is the divergence of the parallel component of the ion flux.

\section{NUMERICAL RESULTS}

The numerical solution of Eqs $(10,23)$ involves two kinds of difficulties. The first difficulty is associated with the secular behavior of the curvature terms $\left[\Omega_{\mathrm{di}}\right.$ in the ion gyro-kinetic equation (10); and $S_{\perp}$ in the electron density equation (23)]. In the limit of large toroidal angle, it can be shown that these quantities scale linearly with $\bar{\zeta}$. Therefore, if the modes have 
a broad extent along the magnetic field line (as one would expect in a low-shear system), the secular terms will be become important and the numerical integration then requires a small time step. The second difficulty is associated with the different characteristic time scales of the ion and electron dynamics. In particular the characteristic time scale associated with the electron dynamics, Eq. (23), is small because the transport along the field line is large [i.e. $\xi_{\mathrm{c}} \gg 1$ in Eq. (23)].

The perturbed ion distribution is stored in a 3-D array $g=g\left(V_{\|}, V_{\perp}, \bar{\zeta}\right)$ at each time step. The perturbed electron density $\widetilde{n}_{\mathrm{e}}=\widetilde{n}_{\mathrm{e}}(\bar{\zeta})$ and the fluctuating electrostatic potential $\widetilde{\Phi}=\widetilde{\Phi}(\bar{\zeta})$ are stored in one-dimensional arrays at each time step. The infinite velocity domain of $V_{\|}$and $V_{\perp}$ are approximated by large finite domains. Because of the secular behavior in $\Omega_{\mathrm{di}}$, we use an implicit method for the left-hand side of Eq. (10) and for the term $\Omega_{\mathrm{di}} g$ on the right-hand side. This method requires no pivoting and is time-efficient to integrate Eq. (10) everywhere in velocity space. For the electron dynamics, Eq. (23), the fact that the collisional parameter $\xi_{\mathrm{c}}$ is large requires the use of a fully-implicit method (Appendix B).

For the simulations reported in this paper, the grid in $V_{\|}$ranges from $V_{\| \min }=-12.0$ to $V_{\| \max }=+12.0$ with $N_{\|}=260$ grid points; the grid in $V_{\perp}$ ranges from 0 to $V_{\perp \max }=7.0$ with $N_{\perp}=120$ grid points. The mesh size in the toroidal angle is $\Delta \bar{\zeta}=\pi / 100$ and the normalized time step is $\Delta t^{\prime \prime}=\omega_{\star} \Delta t=5 \times 10^{-3}$. These parameters have been varied to test their sensitivities. For simplicity, we have set the radial mode number $\theta_{k}$ to zero [the choice $\theta_{k}=0$ is motivated by a recent numerical analysis in a low-shear system ${ }^{22}$. At the beginning of the simulations, we assume that $g$ is Maxwellian with a small amplitude $A(\bar{\zeta})=A_{0} \exp \left[-\sigma\left(\bar{\zeta}-\zeta_{0}\right)^{2}\right]$ where $A_{0} \ll 1$ and $\sigma>0$; we then evaluate $\overline{\bar{n}}$ using Eq. (21). Assuming an adiabatic electron response (only at $t=0$ !), we determine $\widetilde{\Phi}$ along the field line using Eq. (22). The numerical integration of the model Eqs (10) and (23) [coupled through the quasineutrality condition (20)] then proceeds as follows 
- STEP 1 Calculate $g\left(V_{\|}, V_{\perp}, \bar{\zeta}\right)$ everywhere in phase space using Eq. (10) ;

- STEP 2 Calculate $\tilde{n}_{\mathrm{i}}$ using Eq. (19) which is then substituted in the right-hand side of Eq. (23) ;

- STEP 3 Substitute the new value of $\tilde{n}_{\mathrm{e}}$, calculated from Eq. (23), into the right-hand side of Eq. (20) and calculate $\widetilde{\Phi}$;

- STEP 4 Go to STEP 1; [Repeat steps 1-4 until $\left|\partial \gamma / \partial t^{\prime \prime}\right|<\bar{\epsilon}$ and $\left|\partial \omega_{\mathrm{r}} / \partial t^{\prime \prime}\right|<\bar{\epsilon}$, where $\bar{\epsilon} \ll 1$ is a prescribed smallness parameter. See Eqs (24) and (25) for definitions of $\gamma$ and $\left.\omega_{\mathrm{r}}\right]$.

The linear growth rate, $\gamma$, and the mode frequency, $\omega_{\mathrm{r}}$, are calculated dynamically according to

$$
\gamma\left(t^{\prime \prime}\right)=\frac{1}{\left|\langle\hat{\Phi}\rangle_{\zeta}\right|} \frac{\partial\left|\langle\widehat{\Phi}\rangle_{\zeta}\right|}{\partial t^{\prime \prime}}
$$

and

$$
\omega_{\mathrm{r}}\left(t^{\prime \prime}\right)=\Re\left(\frac{i}{\langle\widehat{\Phi}\rangle_{\zeta}} \frac{\partial\langle\widehat{\Phi}\rangle_{\zeta}}{\partial t^{\prime \prime}}\right)
$$

Here $|\Phi| \equiv\left(\Phi \Phi^{\star}\right)^{1 / 2}$ denotes the norm of $\Phi,\langle\widehat{\Phi}\rangle_{\zeta}$ denotes an average along the magnetic field line

$$
\langle\widehat{\Phi}\rangle_{\zeta} \equiv \frac{1}{2 \zeta_{\mathrm{m}}} \int_{\zeta_{0}-\zeta_{\mathrm{m}}}^{\zeta_{0}+\zeta_{\mathrm{m}}} \widehat{\Phi}\left(\zeta^{\prime}\right) d \zeta^{\prime}
$$

where $\Re(X)$ is the real part of $X, \zeta_{0}$ represents a toroidal angle of reference and $\zeta_{\mathrm{m}}$ is a parameter. To determine the correct mode frequency and growth rate, the parameter $\zeta_{\mathrm{m}}$ must be varied until $\omega_{\mathrm{r}}$ and $\gamma$ become independent of $\zeta_{\mathrm{m}}$. In practise, the initial guess for $\zeta_{\mathrm{m}}$ is obtained by estimating the extended toroidal angle at which the secular part of $|\nabla \alpha|$ dominates over its periodic part. The final values for $\omega_{\mathrm{r}}$ and $\gamma$ are obtained when $\left|\partial \omega_{\mathrm{r}} / \partial t^{\prime \prime}\right|$ and $\left|\partial \gamma / \partial t^{\prime \prime}\right|$ become smaller than a prescribed smallness parameter $\bar{\epsilon}$. In this 
paper, we have chosen $\bar{\epsilon}=10^{-3}$. The model equations (10) and (23) have been solved numerically for one field line $\left(\alpha_{0}=0\right.$, which corresponds to the outboard side of the plasma; and $\rho_{0}=\sqrt{s_{0}} \bar{a}=0.95 \bar{a}$ ) of the toroidal heliac H1-NF${ }^{23}$. The 3-D equilibrium state has been computed using the equilibrium code $\mathrm{VMEC}^{24-26}$ for a set of 100 magnetic surface with zero net toroidal current within each flux tube, a volume-averaged $\bar{\beta}$ of $0.36 \%$ and a pressure profile of the form $p_{0}(s)=p_{0}(0)(1-s)^{2}$. In this paper, we retain all harmonics in the equilibrium quantities; however it has recently been suggested ${ }^{27}$ that, for a specific resistive drift-wave model $^{28}$, not all harmonics in the equilibrium are important as far as linear calculations are concerned. Since the VMEC coordinates are not straight, a mapping code is used to specify the equilibrium in straight-field-line coordinates; the geometrical quantities $B(\bar{\zeta})$, $S_{\perp}(\bar{\zeta}), k_{\perp}(\bar{\zeta}),|\nabla \alpha|^{2}(\bar{\zeta})$ and $S_{\|}(\bar{\zeta})$ are calculated using standard curvilinear techniques ${ }^{29}$

In order to get physical insight it is convenient to use advanced visualization techniques ${ }^{29}$ and to display equilibrium quantities that enter the model equations in physical space. In Fig. 1, the magnetic field strength $B$ on the magnetic surface $s_{0}=0.9$ of H1-NF ${ }^{23}$ is shown. The rapid variation of $B$ on the surface is due to the combined effects of the usual $1 / R$ (where $R$ is the distance from the axis of revolution to a point on the surface) and the local modulation due to the toroidal field coils ('patches' in Fig. 1). The normal component of the magnetic curvature $\kappa_{n} \equiv \widehat{\mathbf{n}} \cdot \boldsymbol{\kappa}\left[\right.$ where $\widehat{\mathbf{n}} \equiv(\boldsymbol{\nabla} \psi \cdot \boldsymbol{\nabla} \psi)^{-1 / 2} \boldsymbol{\nabla} \psi$ is the unit vector normal to the magnetic surface, and $\boldsymbol{\kappa} \equiv \widehat{\mathbf{e}}_{\|} \cdot \boldsymbol{\nabla} \widehat{\mathbf{e}}_{\|}$is the magnetic curvature] for the same magnetic surface as in Fig. 1, is shown in Fig. 2. The normal curvature is unfavorable $\left(\kappa_{n}<0\right)$ in the outboard side of the plasma. The geodesic curvature $\kappa_{g} \equiv \widehat{\mathbf{b}} \cdot \boldsymbol{\kappa}$, where $\widehat{\mathbf{b}} \equiv \mathbf{B} \times \widehat{\mathbf{n}} / B$ is the binormal unit vector, is shown in Fig. 3. As in the tokamak case (for which $\kappa_{g} \propto \sin \theta$ ) the geodesic curvature is small in the outboard side of the plasma. As it is evident in Figs 2 and 3 , the main differences in $\kappa_{n}$ and $\kappa_{g}$ between a low- $\beta$ tokamak plasma and a heliac-type plasma arise because of the additional local modulations due to the variation of $R$ (helical axis) and the toroidal field coils. The curvature term in the ballooning representation, $S_{\perp}(\bar{\zeta})$, as function of the extended toroidal angle is shown in Fig. 4. A rapid modulation 
(compare Figs 1-3) along the field line is superimposed to the (asymptotic) linear behavior of $S_{\perp}$. From Fig. 4, one would expect a jagged behavior for the eigenfunction $\widehat{\Phi}$; however, depending on the equilibrium parameters, the parallel transport terms tend to smooth out the rapid variation along the field line ${ }^{28}$. As it turns out, the typical eigenfunction is fairly extended along the field line, with a basic gaussian shape in $\bar{\zeta}$ : the behavior of the eigenfunctions at the end of the simulations is indeed very similar to those reported in a fully-fluid model ${ }^{28}$. In the present study, the rapid modulation due to the toroidal field coil ripples midly modifies the growth rate of the ITG mode. In the context of trapped-particle instabilities, however, the influence of the toroidal field coil ripples on the linear growth rate can be expected to be much more important. The field line of reference in Fig. 4 is $\theta_{0}=\zeta_{0}=0$, which corresponds to a symmetry point in real space; each scalar equilibrium quantity $F_{0}$ should be symmetric in $\bar{\zeta}, F_{0}(-\bar{\zeta})=F_{0}(+\bar{\zeta})$. The parameters (representative of H1-NF experimental conditions) used in the simulations are: $T_{\mathrm{e}}=12 \mathrm{eV}, \tau=2.8$, $n_{0}=6.5 \times 10^{12} \mathrm{~cm}^{-3}, L_{n}=8.0 \mathrm{~cm}$. Fig. 5 shows the asymptotic $\left(\partial / \partial t^{\prime \prime} \approx 0\right)$ growth rate, normalized to $\omega_{\star}$, as a function of $k_{\theta} \rho_{s 0} \propto N$. The ion temperature gradient parameter is $\eta_{\mathrm{i}}=1.9$. The fastest linear growth rate is $k_{\theta} \rho_{s 0} \simeq 0.6$. The corresponding mode frequency, $\omega_{\mathrm{r}}$, is shown in Fig. 6. The mode rotates in the ion diamagnetic direction, and becomes more negative as the toroidal mode number increases (as in the tokamak case). The mode frequency varies linearly between $k_{\theta} \rho_{s 0}=0.4$ and $k_{\theta} \rho_{s 0}=0.8$, and becomes independent of the toroidal mode number for $k_{\theta} \rho_{s 0}>0.8$. We have studied investigated the influence of the ion temperature gradient parameter $\left(\eta_{\mathrm{i}}\right)$ (Fig. 7). The toroidal mode number is unchanged at $k_{\theta} \rho_{s 0}=1.0$. The mode is (linearly) stable for small $\eta_{\mathrm{i}}$; the threshold for instability occurs at $\eta_{\mathrm{i}}=\eta_{\mathrm{ic}} \simeq 1.12$. For $\eta_{\mathrm{i}}>\eta_{\mathrm{ic}}$, the linear growth rate scales approximately linearly with the ion temperature gradient parameter. Although the geometrical details (in the ballooning representation) between a tokamak with circular magnetic surfaces and a fully 3-D stellarator with a helical magnetic axis are substantially different, the linear growth rate and mode frequency are qualitatively quite similar. 


\section{CONCLUSION}

We have studied low-frequency, drift-type modes in the geometry of a three-field period stellarator. In the low-frequency regime, the plasma is quasineutral

$$
\widetilde{n}_{\mathrm{i}}(\widetilde{\Phi})=\widetilde{n}_{\mathrm{e}}(\widetilde{\Phi})
$$

The perturbed ion density, $\widetilde{n}_{\mathrm{i}}$, has been calculated by taking the velocity moment of the ion distribution function $\delta f$ which, in turn, has been obtained by solving the linear, collisionless ion gyro-kinetic equation in the ballooning representation. The fluctuating electron density, $\widetilde{n}_{\mathrm{e}}$, has been calculated using Braginskii's fluid equations. Because of the fast electron transport along B, an implicit method has been used for the electron dynamics. Numerical simulations have been carried out for a low-shear, three-field-period stellarator. The fastest (linear) growth rate, $\gamma$, increases with the toroidal mode number $N$; for very large $N$, however, the growth rate decreases with $N$. A scan in the ion temperature gradient parameter $\eta_{\mathrm{i}}$ showed that a drift mode becomes unstable for $\eta_{\mathrm{i}}>\eta_{\mathrm{ic}} \simeq 1.12$. The $\eta_{\mathrm{i}}$ threshold found in this study is comparable to $\eta_{\text {ic }}$ found in tokamaks with similar equilibrium parameters. Although the curvature term in the ballooning representation has a jagged behavior (Fig. 4) as a result of the rapid modulation of equilibrium quantities on the magnetic surface (Figs 1-3), the qualitative dependence of the growth rate on the toroidal mode number (Fig. 5) and on the $\eta_{\text {i }}$ parameter (Fig. 7) is quite similar to what can be expected in a medium-sized tokamak plasma.

A limitation of our model is the neglect of trapped particles. As the plasma collisionality decreases, the appearance of toroidally-trapped and helically-trapped particles can lead to new instabilities ${ }^{17}$. Inclusion of magnetic trapping in stellarator geometry is left for future work. Although a linear formalism can help to provide physical insight, fully three-dimensional, nonlinear turbulence simulations, including the effects of zonal flows ${ }^{30}$, are required to make comparison between stellarator and tokamak transport. Recent nonlinear fluid simulations 
of drift-wave turbulence in the flux-tube geometry by Kendl et al. ${ }^{31}$ represent a noticeable step towards that goal. 


\section{APPENDIX A: ELECTRON FLUID MODEL}

The starting equations are the electron continuity equation ${ }^{8}$

$$
\frac{\partial n_{\mathrm{e}}}{\partial t}+\boldsymbol{\nabla} \cdot\left(n_{\mathrm{e}} \mathbf{V}_{\mathrm{e}}\right)=0
$$

and the electron momentum equation (neglecting the electron inertia and stress tensor)

$$
e n_{\mathrm{e}}\left(\mathbf{E}+\frac{\mathbf{V}_{\mathrm{e}} \times \mathbf{B}}{c}\right)+\nabla p_{\mathrm{e}}=\mathbf{R}_{\mathrm{ei}},
$$

where $\mathbf{V}_{\mathrm{e}}$ is the electron fluid velocity, $p_{\mathrm{e}}=n_{\mathrm{e}} T_{\mathrm{e}}$ is the electron pressure and

$$
\mathbf{R}_{\mathrm{ei}}=\eta_{\|} \mathbf{J}_{\|}+\eta_{\perp} \mathbf{J}_{\perp}-0.71 n_{\mathrm{e}} \boldsymbol{\nabla}_{\|} T_{\mathrm{e}}-\frac{3 n_{\mathrm{e}}}{2 \omega_{c \mathrm{e}} \tau_{\mathrm{e}}} \widehat{\mathbf{e}}_{\|} \times \nabla T_{\mathrm{e}} .
$$

is the friction force arising from electron-ion collisions for a plasma with singly-charged ions ${ }^{8}$ ( $\mu=0.71$ is a thermoelectric coefficient). Here $\eta_{\|}$and $\eta_{\perp}$ are the parallel and perpendicular classical resistivities, respectively; $\omega_{c e} \equiv(e B) /\left(m_{\mathrm{e}} c\right)$ is the electron cyclotron frequency; and $\tau_{\mathrm{e}}$ is the electron basic collisional time. In the low-frequency regime, one can solve Eq. (A2) perturbatively for $\mathbf{V}_{\mathrm{e} \perp}$

$$
\mathbf{V}_{\mathrm{e} \perp}=\mathbf{V}_{E}+\mathbf{V}_{\mathrm{de}}+\mathbf{V}_{c},
$$

where

$$
\begin{aligned}
\mathbf{V}_{E} & \equiv \frac{c}{B} \mathbf{E} \times \widehat{\mathbf{e}}_{\|}, \\
\mathbf{V}_{d \mathrm{e}} & \equiv \frac{c}{e n_{\mathrm{e}} B} \nabla p_{\mathrm{e}} \times \widehat{\mathbf{e}}_{\|}, \\
\mathbf{V}_{c} & \equiv \frac{c}{e n_{\mathrm{e}} B} \widehat{\mathbf{e}}_{\|} \times \mathbf{R}_{\mathrm{ei}},
\end{aligned}
$$

are the lowest-order $\mathbf{E} \times \mathbf{B}$ drift velocity, the electron diamagnetic drift velocity and the ion-electron-collision-driven drift velocity, respectively. In the linear approximation, the divergence of the perpendicular electron flux is

$$
\boldsymbol{\nabla} \cdot\left(n_{\mathrm{e}} \mathbf{V}_{\mathrm{e} \perp}\right)=\underbrace{\mathbf{V}_{E} \cdot \boldsymbol{\nabla} n_{0}}_{[\mathbf{1}]}+\underbrace{n_{0} \boldsymbol{\nabla} \cdot \mathbf{V}_{E}+\boldsymbol{\nabla} \cdot\left(n_{\mathrm{e}} \mathbf{V}_{\mathrm{de}}\right)}_{[\mathbf{2}]}+\underbrace{\boldsymbol{\nabla} \cdot\left(\frac{c}{e B^{2}} \mathbf{B} \times \mathbf{R}_{\mathrm{ei}}\right)}_{[3]},
$$


where term [1] represents the linear drive, term [2] are curvature contributions, and term [3], which arises due to the perpendicular resistivity, is roughly $\left(\omega_{\text {ce }} \tau_{\mathrm{e}}\right)^{-1} \ll 1$ times smaller than term [1]. For any scalar $f$, we note that

$$
\begin{aligned}
\boldsymbol{\nabla} \cdot\left(\frac{\mathbf{B} \times \boldsymbol{\nabla f}}{B^{2}}\right) & =\boldsymbol{\nabla f}^{\boldsymbol{\nabla} \times\left(\frac{\mathbf{B}}{B^{2}}\right)}-\frac{\mathbf{B}}{B^{2}} \cdot \boldsymbol{\nabla} \times \boldsymbol{\nabla f} \\
& =\underbrace{\frac{\boldsymbol{\nabla} f}{B^{2}} \cdot(\boldsymbol{\nabla} \times \mathbf{B})}_{[\mathbf{1}]}+\underbrace{2 \boldsymbol{\nabla} f \cdot\left(\widehat{\mathbf{e}}_{\|} \times \frac{\boldsymbol{\nabla} B}{B^{2}}\right)}_{[\mathbf{2}]} .
\end{aligned}
$$

From Ampere's law, $4 \pi \mathbf{J}_{0}=c \boldsymbol{\nabla} \times \mathbf{B}$ (where $\mathbf{J}_{0}$ is the equilibrium current density) and the radial force balance, $\mathbf{J}_{0} \times \nabla p_{0}=c \boldsymbol{\nabla} p_{0}$, it follows that $|\boldsymbol{\nabla} \times \mathbf{B}| / B \sim \beta / L_{n}$, where $\beta \equiv 8 \pi p_{0} / B_{0}^{2}$. Noting that $|\nabla B / B| \sim 1 / \bar{R}(\bar{R}$ being the average radius of the magnetic axis), it follows that the ratio of the two terms on the right-hand side of Eq. (A7) is $($ term $[\mathbf{1}]) /(\operatorname{term}[\mathbf{2}]) \sim \beta /\left(2 \epsilon_{n}\right)$, where $\epsilon_{n} \equiv L_{n} / \bar{R}$ is the toroidicity parameter. Below we shall assume that $\beta \ll \epsilon_{n}$. The compression of the lowest-order $\mathbf{E} \times \mathbf{B}$ drift velocity reads [neglecting corrections of $\mathcal{O}(\beta)]$

$$
\boldsymbol{\nabla} \cdot \mathbf{V}_{E} \simeq 2 \frac{c T_{\mathrm{e} 0}}{e B_{0} L_{n}} \nabla \widetilde{\Phi} \cdot\left(\frac{\widehat{\mathbf{e}}_{\|} \times \mathbf{Q}}{B_{\star}}\right)=2 i \omega_{\star} \sqrt{b_{0}} S_{\perp}(\bar{\zeta}) \widetilde{\Phi}
$$

where, as before, $\mathbf{Q} \equiv L_{n} \nabla B / B=\mathcal{O}\left(\epsilon_{n}\right)$ is related to the magnetic curvature, $B_{\star}(\bar{\zeta}) \equiv B(\bar{\zeta}) / B_{0}$ is the normalized magnetic field strength and $S_{\perp}(\bar{\zeta})$ is defined in Eq. (15). We note that the $\mathbf{E} \times \mathbf{B}$ drift velocity in the ballooning representation reads $\mathbf{V}_{E}=i c_{s} \sqrt{b_{0}}\left(\widehat{\mathbf{e}}_{\|} \times \widehat{\mathbf{e}}_{\perp} / B_{\star}\right) \widetilde{\Phi}$ so that the linear drive is simply $\mathbf{V}_{E} \cdot \nabla n_{0}=-i n_{0} \omega_{\star} \sqrt{b_{0}} \widetilde{\Phi}$, where we used the relation $B_{\star}^{-1} \nabla \rho \cdot\left(\widehat{\mathbf{e}}_{\|} \times \widehat{\mathbf{e}}_{\perp}\right)=1$. We again use Eq. (A7), neglect finite- $\beta$ corrections, to obtain the divergence of the electron diamagnetic flux

$$
\boldsymbol{\nabla} \cdot\left(n_{\mathrm{e}} \mathbf{V}_{\mathrm{de}}\right)=-2 i n_{0} \omega_{\star} \sqrt{b_{0}} S_{\perp}(\bar{\zeta})\left(\tilde{n}_{\mathrm{e}}+\widetilde{T}_{\mathrm{e}}\right)
$$

Combining the above results one obtains the divergence of the cross-field electron flux

$$
\boldsymbol{\nabla} \cdot \Gamma_{\mathrm{e} \perp}=-i \Gamma_{\star} \sqrt{b_{0}}\left[\widetilde{\Phi}+2 S_{\perp}(\widetilde{\zeta})\left(\widetilde{h}+\widetilde{T}_{\mathrm{e}}\right)\right]
$$

where $\boldsymbol{\Gamma}_{\mathrm{e} \perp} \equiv n_{\mathrm{e}} \mathbf{V}_{\mathrm{e} \perp}, \Gamma_{\star} \equiv n_{0} \omega_{\star}=n_{0} c_{s} / L_{n}$ and $h(\bar{\zeta})=\widetilde{n}_{\mathrm{e}}(\bar{\zeta})-\widetilde{\Phi}(\bar{\zeta})$ is the nonadiabatic part of the electron response. Taking the scalar product of the electron momentum equation (A2) with $\widehat{\mathbf{e}}_{\|}$and solving for $J_{\|}$ 


$$
J_{\|}=e n_{0} c_{s} \xi_{\mathrm{c}} L_{n} \nabla_{\|}\left[\widetilde{h}+(1+\mu) \widetilde{T}_{\mathrm{e}}\right]
$$

where $\xi_{\mathrm{c}} \equiv 2\left(\omega_{\star} \tau_{\mathrm{e}}\right)\left(m_{\mathrm{i}} / m_{\mathrm{e}}\right) \gg 1$ is termed the 'collisional parameter'. Noting that

$$
\boldsymbol{\nabla} \cdot \mathbf{J}_{\|}=\nabla_{\|} J_{\|}+J_{\|} \boldsymbol{\nabla} \cdot \widehat{\mathbf{e}}_{\|}=\nabla_{\|} J_{\|}-J_{\|} Q_{\|}(\bar{\zeta}) / L_{n}
$$

and defining $\widetilde{G} \equiv \widetilde{h}+(1+\mu) \widetilde{T}_{\mathrm{e}}$, one gets the divergence of the parallel current density

$$
\nabla \cdot \mathbf{J}_{\|}=e \Gamma_{\star} \xi_{\mathrm{c}}\left[L_{n}^{2} \nabla_{\|}^{2} \widetilde{G}-Q_{\|}(\bar{\zeta}) \nabla_{\|} \widetilde{G}\right]
$$

Combining Eqs (A10,A13) in the electron continuity equation (A1) one gets

$$
\frac{\partial \widetilde{n}_{\mathrm{e}}}{\partial t^{\prime \prime}}=\xi_{\mathrm{c}}\left[L_{n}^{2} \nabla_{\|}^{2} \widetilde{G}-Q_{\|}(\bar{\zeta}) L_{n} \nabla_{\|} \widetilde{G}\right]+i \sqrt{b_{0}}\left[\widetilde{\Phi}+2 S_{\perp}(\bar{\zeta})\left(\widetilde{h}+\widetilde{T}_{\mathrm{e}}\right)\right]-S_{\mathrm{i}}^{(\|)}
$$

where, as before, $t^{\prime \prime} \equiv \omega_{\star} t$ is the normalized time and $S_{\mathrm{i}}^{(||)} \equiv \boldsymbol{\nabla} \cdot \boldsymbol{\Gamma}_{\mathrm{i} \|} /\left(n_{0} \omega_{\star}\right)$. If the electron temperature fluctuations are neglected, $\widetilde{T}_{\mathrm{e}} \mapsto 0$, then $\widetilde{G} \mapsto \widetilde{h}$ and

$$
\frac{\partial \widetilde{n}_{\mathrm{e}}}{\partial t^{\prime \prime}}=\xi_{\mathrm{c}}\left[L_{n}^{2} \nabla_{\|}^{2} \widetilde{h}-Q_{\|}(\bar{\zeta}) L_{n} \nabla_{\|} \widetilde{h}\right]+i \sqrt{b_{0}}\left[\widetilde{\Phi}+2 S_{\perp}(\bar{\zeta}) \widetilde{h}\right]-S_{\mathrm{i}}^{(\|)}
$$

To calculate the term related to the parallel ion dynamics, $S_{\mathrm{i}}^{(\mid l)}$, we use

$$
\delta f=F_{M}\left[g J_{0}+\frac{\widetilde{\Phi}}{\tau}\left(J_{0}^{2}-1\right)\right]
$$

and

$$
\int \frac{d^{3} v}{n_{0}} F_{M}=\frac{2}{\sqrt{\pi}} \int_{0}^{\infty} d V_{\perp} \int_{-\infty}^{+\infty} d V_{\|} V_{\perp} \exp \left(-V_{\|}^{2}-V_{\perp}^{2}\right)
$$

We may write

$$
S_{\mathrm{i}}^{(\|)}=-Q_{\|}(\bar{\zeta}) \frac{V_{\|}^{(\mathrm{f})}}{c_{s}}+L_{n} \nabla_{\|} \frac{V_{\|}^{(\mathrm{f})}}{c_{s}}
$$

where the parallel component of the fluid ion velocity can be written as

$$
V_{\|}^{(\mathrm{f})}=\frac{1}{n_{0}} \int d^{3} v v_{\|} \delta f .
$$

Collecting equations (A16,A17,A19) we find that

$$
\frac{V_{\|}^{(\mathrm{f})}}{c_{s}}=\frac{\sqrt{8 \tau}}{\sqrt{\pi}} \int_{0}^{\infty} d V_{\perp} \int_{-\infty}^{+\infty} d V_{\|} V_{\perp} V_{\|} H\left(V_{\|}, V_{\perp}, \bar{\zeta}\right) \exp \left(-V_{\|}^{2}-V_{\perp}^{2}\right),
$$

where

$$
H\left(V_{\|}, V_{\perp}, \bar{\zeta}\right) \equiv J_{0}\left(V_{\perp}, \bar{\zeta}\right) g\left(V_{\|}, V_{\perp}, \bar{\zeta}\right)+\frac{\widetilde{\Phi}(\bar{\zeta})}{\tau}\left[J_{0}^{2}\left(V_{\perp}, \bar{\zeta}\right)-1\right]
$$




\section{APPENDIX B: NUMERICAL METHOD}

With a slight change of notation, $S_{i}^{\|} \mapsto S, \widetilde{n}_{\mathrm{e}} \mapsto N, t^{\prime \prime} \mapsto t, \widetilde{\Phi} \mapsto \Phi$ and $\widetilde{h} \mapsto h \equiv N-\Phi$, we must solve

$$
\frac{\partial N}{\partial t}=\xi_{\mathrm{c}}\left(L_{n}^{2} \nabla_{\|}^{2} h-Q_{\|} L_{n} \nabla_{\|} h\right)+i \sqrt{b_{0}}\left(\Phi+2 S_{\perp} h\right)-S
$$

where

$$
\nabla_{\|}=\frac{S_{\|}}{\bar{R}} \frac{\partial}{\partial \bar{\zeta}}
$$

is the parallel gradient operator (keeping $\alpha$ constant), and $S_{\|} \sim 1$ varies along the field line [In a tokamak with circular, concentric magnetic surfaces, $\bar{\zeta} \mapsto q \bar{\theta}$ (where $\bar{\theta}$ is the extended poloidal angle), $S_{\|}=1$ and the parallel gradient operator assumes its usual form $\left.\nabla_{\|}=\left(q R_{0}\right)^{-1} \partial / \partial \bar{\theta}\right]$. Upon substituting Eq.(B2) in Eq.(B1) and re-arranging the terms, one gets

$$
\frac{\partial N}{\partial t}-\xi_{\mathrm{c}} \epsilon_{n}^{2} S_{\|}^{2} \frac{\partial^{2} N}{\partial \bar{\zeta}^{2}}+\xi_{\mathrm{c}} \epsilon_{n} S_{\|} G \frac{\partial N}{\partial \bar{\zeta}}-2 i \sqrt{b_{0}} S_{\perp} N=R,
$$

where $S_{\|}, S_{\perp}, G \equiv Q_{\|}-\epsilon_{n} d S_{\|} / d \zeta$ and

$$
R(\bar{\zeta}, t) \equiv i \sqrt{b_{0}} \Phi\left(1-2 S_{\perp}\right)-S-\xi_{\mathrm{c}} \epsilon_{n}^{2} S_{\|}^{2} \frac{\partial^{2} \Phi}{\partial \bar{\zeta}^{2}}+\xi_{\mathrm{c}} \epsilon_{n} S_{\|} G \frac{\partial \Phi}{\partial \bar{\zeta}}
$$

depend on the position along the field line. In Eqs (B3) and (B4), $\epsilon_{n}=L_{n} / \bar{R}$ is the toroidicity parameter. We define discrete grids in $\bar{\zeta}$ and $t$ and let $N_{j}^{(k)} \equiv N\left(\bar{\zeta}_{j}, t_{k}\right)$, where $t_{k+1}-t_{k}=\Delta t=$ time step and $\bar{\zeta}_{j+1}-\bar{\zeta}_{j}=\Delta \bar{\zeta}=$ mesh size. Using an implicit method, the finite difference form of Eq.(B3) reads

$$
\left(\overline{\alpha_{j}}-\overline{\beta_{j}}\right) N_{j+1}^{(k+1)}+\left(1+2 \overline{\beta_{j}}-2 i \sqrt{b_{0}} S_{\perp j} \Delta t\right) N_{j}^{(k+1)}-\left(\overline{\alpha_{j}}+\overline{\beta_{j}}\right) N_{j-1}^{(k+1)}=r_{j}^{(k)}
$$

where

$$
\begin{aligned}
\overline{\alpha_{j}} & =\xi_{\mathrm{c}} \epsilon_{n} \frac{\Delta t}{2 \Delta \bar{\zeta}} S_{\| j} G_{j} \\
\overline{\beta_{j}} & =\xi_{\mathrm{c}} \epsilon_{n}^{2} S_{\| j}^{2} \frac{\Delta t}{(\Delta \bar{\zeta})^{2}} \\
r_{j}^{(k)} & =N_{j}^{(k)}+R_{j}^{(k)} \Delta t .
\end{aligned}
$$


The system of $N_{\bar{\zeta}}$ equations defined by Eq.(B5) is written as a tridiagonal matrix, which is solved using the standard LU decomposition method. 


\section{REFERENCES}

${ }^{1}$ W. M. Tang, Nucl. Fusion 18, 1089 (1978).

${ }^{2}$ P. C. Liewer, Nucl. Fusion 25, 543 (1985).

${ }^{3}$ W. Horton, Rev. Mod. Phys. 71, 735 (1999).

${ }^{4}$ W. Horton, Phys. Fluids B1(3), 524 (1989).

${ }^{5}$ A. Bhattacharjee, J. E. Sedlak, P. L. Similon, M. N. Rosenbluth and D. W. Ross, Phys. Fluids 26(4), 880 (1983).

${ }^{6}$ M. Persson, J. L. V. Lewandowski and H. Nordman, Phys. Plasmas 2(9), 3440 (1996).

${ }^{7}$ N. Dominguez, B. A. Carreras and V. E. Lynch, Phys. Fluids B4 (9), 2894 (1992).

${ }^{8}$ S.I. Braginskii, in Review of Plasma Physics, edited by M.A. Leontovitch (Consultants Bureau, N.Y., 1965).

${ }^{9}$ J. W. Connor, R. J. Hastie and J. B. Taylor, Phys. Rev. Lett. 40(6), 396 (1978).

10 T. M. Antonsen and J. B. Lane, Phys. Fluids 23(6), 1205 (1980).

${ }^{11}$ W. D. D'haeseleer, W. N. G. Hitchon, J.D. Callen and J. L. Shohet, Flux Coordinates and Magnetic Field Structure (Springer, Berlin, 1991).

${ }^{12}$ L. I. Rudakov and R. Z. Sagdeev, Sov. Phys. Dokl. 6(5), 415 (1961).

${ }^{13}$ O. P. Pogutse, Sov. Phys. JETP 25, 498 (1967).

${ }^{14}$ B. Coppi, M. N. Rosenbluth and R. Z. Sagdeev, Phys. Fluids 10, 582 (1967).

${ }^{15}$ A. B. Hassam, T. M. Antonsen, J. F. Drake and P. N. Gudzar, Phys. Fluids B2 (8), 1822 (1990).

${ }^{16}$ W. Horton, D. Choi and W. M. Tang, Phys. Fluids 24(8), 1077 (1981).

${ }^{17}$ B. B. Kadomtsev and O. P. Pogutse, Nucl. Fusion 11, 67 (1971). 
${ }^{18}$ B. Coppi and G. Rewoldt, Phys. Rev. Lett. 33(22), 1329 (1974).

${ }^{19}$ W. M. Tang, J. C. Adam and D. W. Ross, Phys. Fluids 20, 430 (1977).

${ }^{20}$ P. H. Rutherford and E. A. Frieman, Phys. Fluids 11(3), 569 (1968).

${ }^{21}$ I.S. Gradshteyn and I.M. Ryzhik, Table of Integrals, Series, and Products, (Academic Press, New York, 1965).

${ }^{22}$ J.L.V. Lewandowski and R.M. Ellem, Aust. J. Phys., 52, 71 (1999).

${ }^{23}$ S. M. Hamberger, B. D. Blackwell, L. E. Sharp and D. B. Shenton, Fusion Tech. 17, 123 (1990)

${ }^{24}$ S.P. Hirshman and J.C. Whitson, Phys. Fluids 26, 3553 (1983).

${ }^{25}$ S.P. Hirshman and H.K. Meier, Phys. Fluids 28, 1387 (1985).

${ }^{26}$ S. P. Hirshman and O. Betancourt, J. Comp. Phys. 86, 99 (1991).

${ }^{27}$ J.L.V. Lewandowski, Phys. Plasmas 6(12), 4788 (1999).

28 J.L.V. Lewandowski, Phys. Plasmas 4(11), 4023 (1997).

29 J.L.V. Lewandowski, J. Res. Phys., 27(2), 67 (1998).

30 J.L.V. Lewandowski, Z. Lin, W.W. Lee and T.S. Hahm, Phys. Plasmas 7 92), 588 (2000).

${ }^{31}$ A. Kendl (private communication, 1999). 
Figure 1 The magnetic field strength (T) on a magnetic surface of the toroidal H1-NF. The radial label of the magnetic surface is $s_{0}=0.90$.

Figure 2 The normal component of the magnetic curvature $\left(m^{-1}\right)$ for the same magnetic surface shown in Fig. 1.

Figure 3 The geodesic component of the magnetic curvature $\left(\mathrm{m}^{-1}\right)$ for the same magnetic surface shown in Fig. 1.

Figure 4 The curvature term $\left(S_{\perp}\right)$ as a function of the extended toroidal angle. The field line of reference is $\theta_{0}=\zeta_{0}=0$.

Figure 5 The normalized growth rate as a function of $k_{\theta} \rho_{s}$ for the field of reference $\theta_{0}=$ $\zeta_{0}=0$.

Figure 6 The real frequency as a function of $k_{\theta} \rho_{s}$ for the field of reference $\theta_{0}=\zeta_{0}=0$.

Figure 7 The normalized growth rate as a function of the ion temperature gradient parameter $\eta_{\mathrm{i}}$ for the same field line as in Figs 4-6. 


\section{FIG.1 Lewandowski}

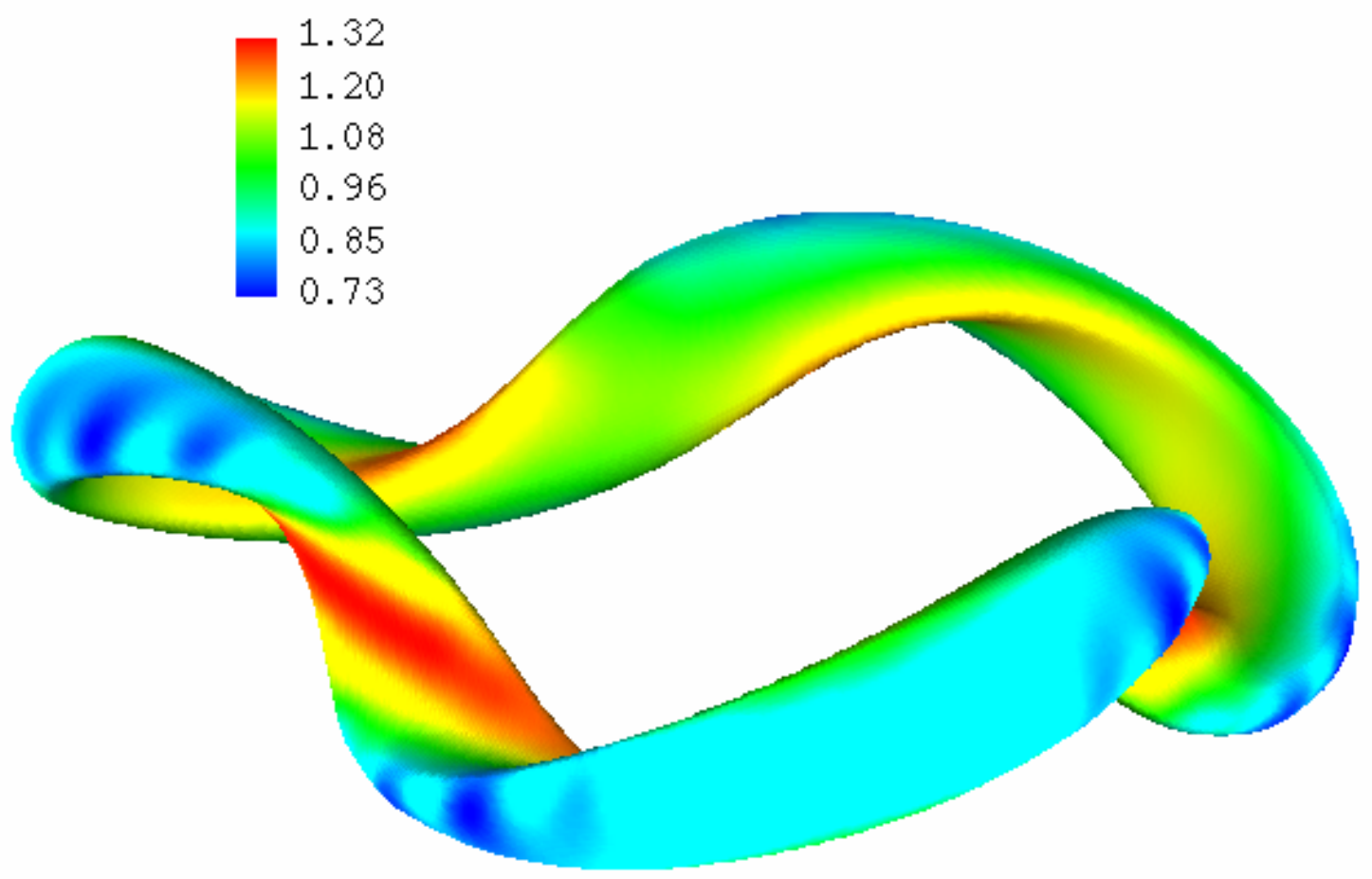




\section{FIG.2 Lewandowski}

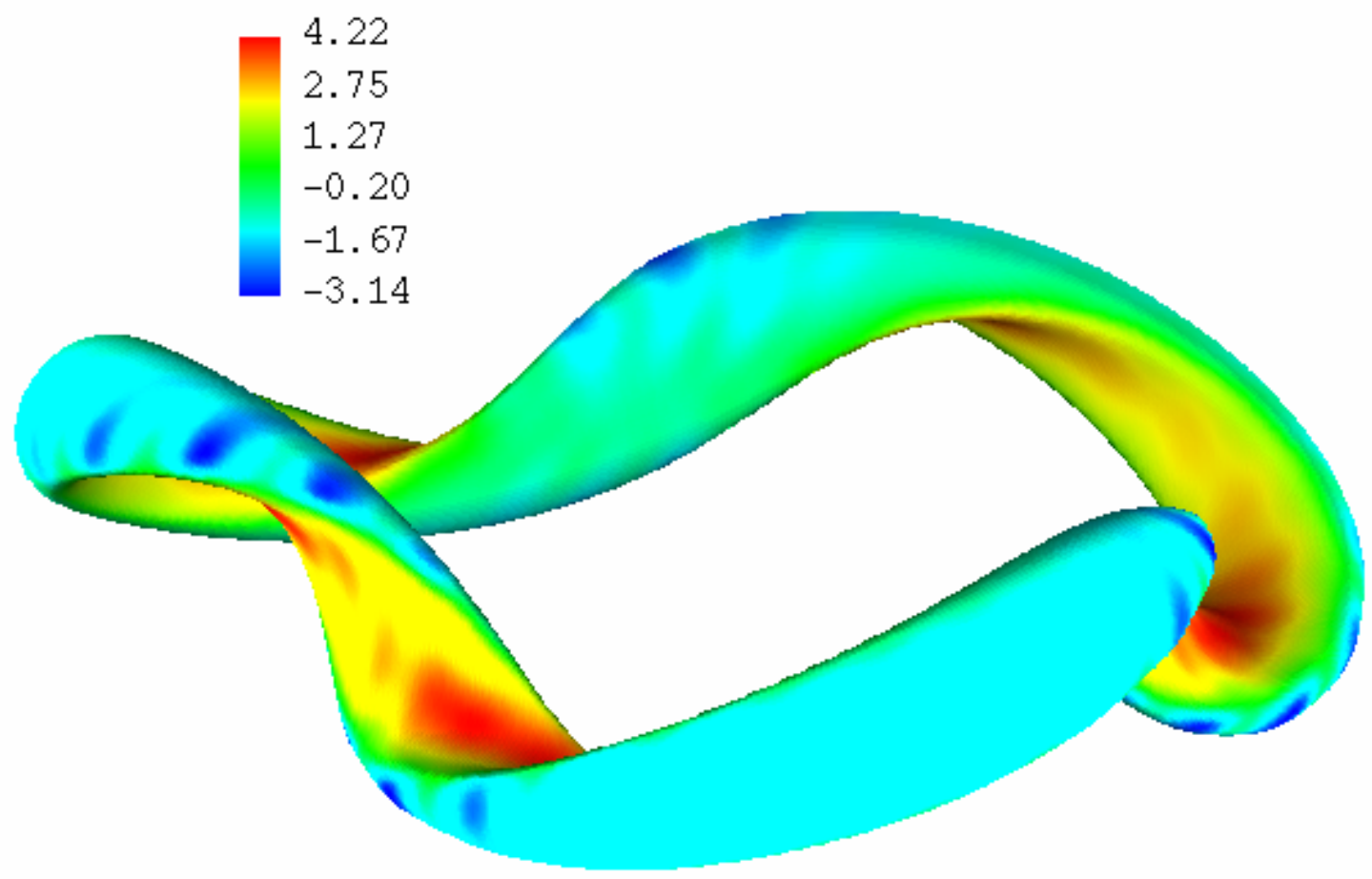




\section{FIG.3 Lewandowski}

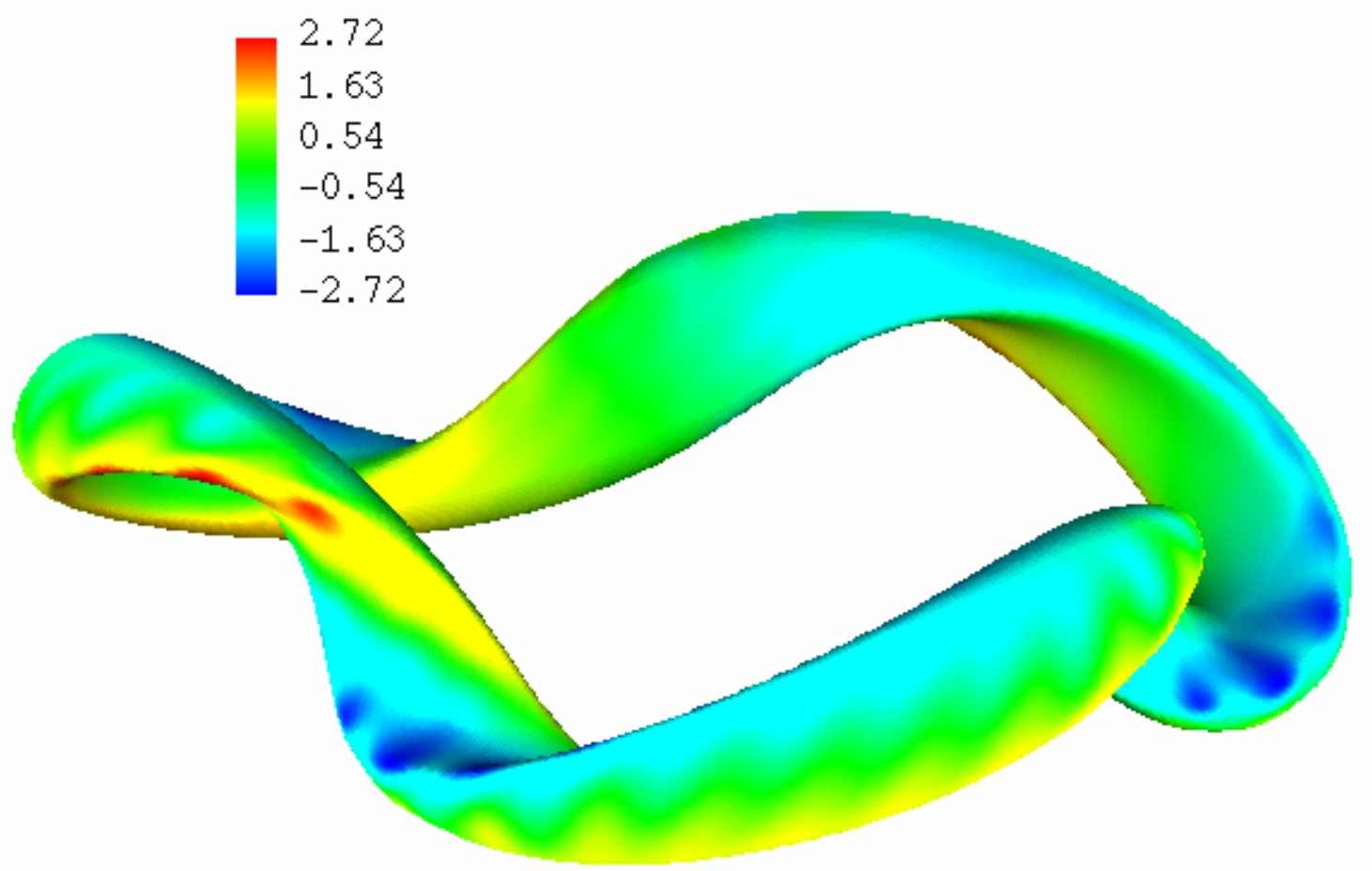


FIG.4 Lewandowski

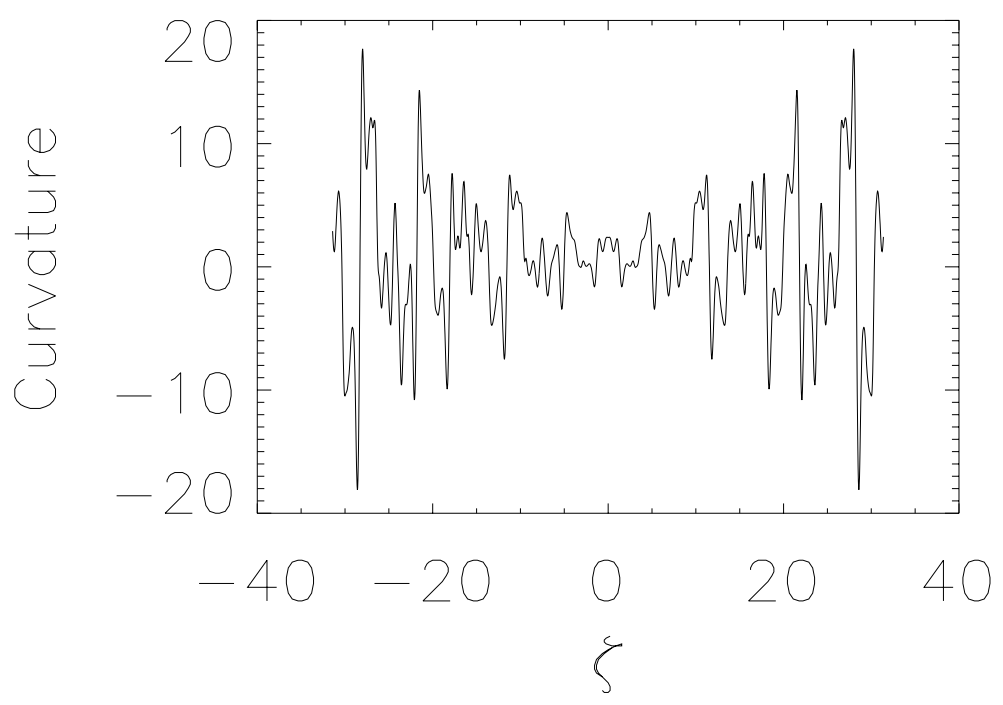




\section{FIG.5 Lewandowski}

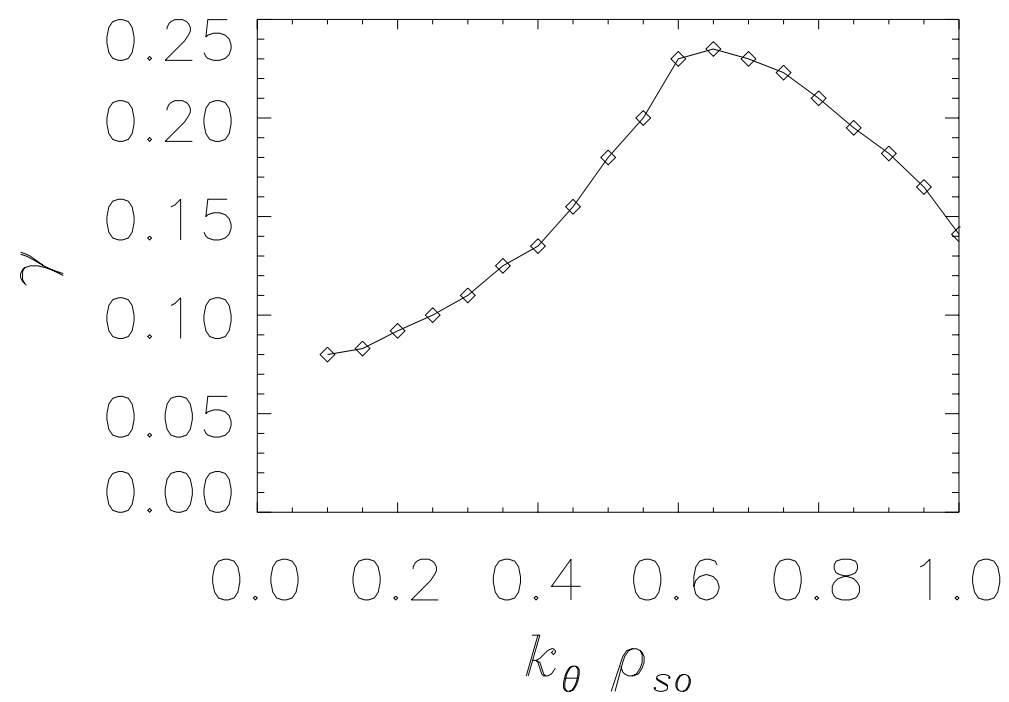




\section{FIG.6 Lewandowski}

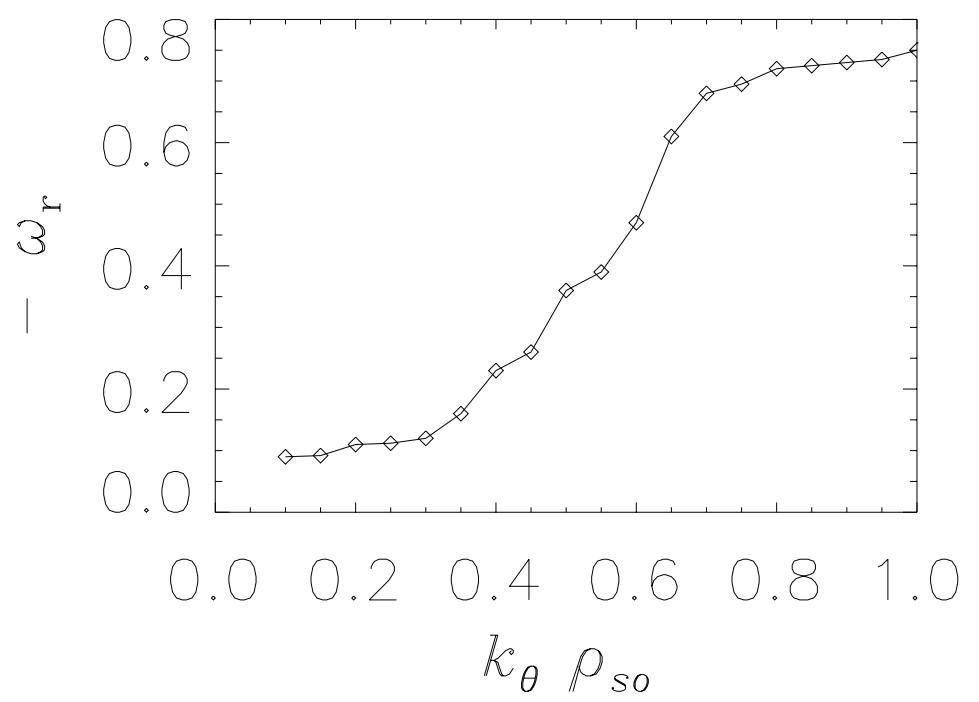




\section{FIG.7 Lewandowski}

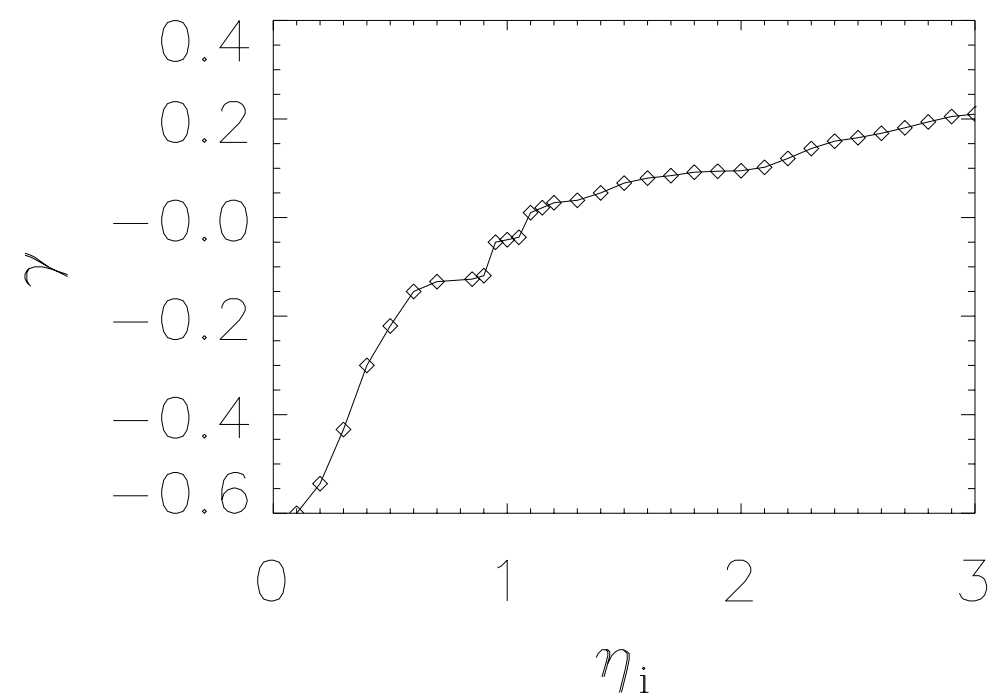

